\title{
Electronic structure and electron-transport properties of three metal hexacyanoferrates
}

\author{
Kevin Hurlbutt, ${ }^{\dagger}$ Feliciano Giustino, ${ }^{\ddagger}$ Mauro Pasta, ${ }^{*}, \dagger$ and George Volonakis ${ }^{*}, \S$ \\ $\dagger$ Department of Materials, University of Oxford, Parks Road, Oxford OX1 3PH, United \\ Kingdom \\ $\ddagger$ Oden Institute for Computational Engineering and Sciences, The University of Texas at \\ Austin, Austin, Texas 78712, United States \\ IDepartment of Physics, The University of Texas at Austin, Austin, Texas 78712, United \\ States \\ $\S$ Univ Rennes, ENSCR, INSA Rennes, CNRS, ISCR (Institut des Sciences Chimiques de \\ Rennes), UMR 6226, Rennes, France
}

E-mail: mauro.pasta@materials.ox.ac.uk; yorgos.volonakis@univ-rennes1.fr

\begin{abstract}
Metal hexacyanometallates, or Prussian blue analogs (PBAs), are active materials in important electrochemical technologies, including next-generation sodium- and potassiumion batteries. They have tunable properties including reduction potential, ionic conductivity, and color. However, little is known about their electronic conductivities. In this work, we use density-functional theory to model electronic structure and to explore the likely electron-conduction mechanism in three promising cathodes (manganese, iron, and cobalt hexacyanoferrate) in each of three oxidation states. First, we demonstrate that hybrid functionals reliably reproduce experimentally observed spin configurations and geometric phase changes. We confirm these materials are semiconductors or insulators with band gaps ranging from $1.90 \mathrm{eV}$ up to $4.94 \mathrm{eV}$. We further identify that for
\end{abstract}


most of the compounds the electronic band edges originate from carbon-coordinatediron orbitals, suggesting that doping at the carbon-coordinated site may strongly affect carrier conductivity. Finally, we calculate charge-carrier effective masses, which we find are very heavy. This study is an important foundation for making electronic conductivity a tunable PBA material property.

\section{Introduction}

The metal hexacyanometallates, known more commonly as the Prussian blue analogs (PBAs), are promising active materials as electrodes for next-generation, sodium-ion batteries. ${ }^{1-3}$ PBAs are also important in potassium-ion ${ }^{4}$ and all-solid-state ${ }^{5}$ batteries, as well as in other electrochemical technologies including electrocatalysis, ${ }^{6}$ thermionics, ${ }^{7}$ and smart windows. ${ }^{8}$ They are a highly substitutable, and therefore tunable, material system. ${ }^{9}$ Their general formula is $\mathrm{A}_{\mathrm{x}} \mathrm{M}^{\mathrm{J}}\left[\mathrm{M}^{\prime \mathrm{K}}(\mathrm{CN})_{6}\right]$, in which $\mathrm{A}$ is an alkali or alkali-earth metal, $\mathrm{M}$ and $\mathrm{M}^{\prime}$ are possibly distinct transition metals in oxidation states $\mathrm{J}$ and $\mathrm{K}$, respectively. Appropriate compositions can be selected that undergo two electrochemical reactions within the stability window of conventional organic electrolytes, for specific capacities around $170 \mathrm{~mA} \mathrm{~h} \mathrm{~g}^{-1}$ at potentials of approximately $+1.0 \mathrm{~V}$ versus the standard hydrogen electrode. ${ }^{10}$ Their impressive rate capabilities and high ionic conductivities ${ }^{11,12}$ derive from the wide channels in their crystal structure, shown in Figure 1, for fast ion movement through the bulk.

This work addresses two conspicuously understudied areas of PBA science: electronic conductivity and first-principles modelling. First, past studies have carefully correlated chemical composition with materials properties including, for example, lattice parameter, ${ }^{13}$ reduction potential, ${ }^{14}$ and coefficient of thermal expansion. ${ }^{15}$ But there has never been a systematic study of the effect of composition on electronic conductivity. This is despite the fact that electronic conductivity is important in virtually every PBA-based technology. Early work on the composition $\mathrm{NaFe}\left[\mathrm{Fe}(\mathrm{CN})_{6}\right]$ (also known as Prussian blue, the prototype compound) reported a value on the order of $\sigma_{\mathrm{e}}=10^{-5} \mathrm{~S} \mathrm{~m}^{-1}$, but it lacked structural characterization. ${ }^{16}$ 


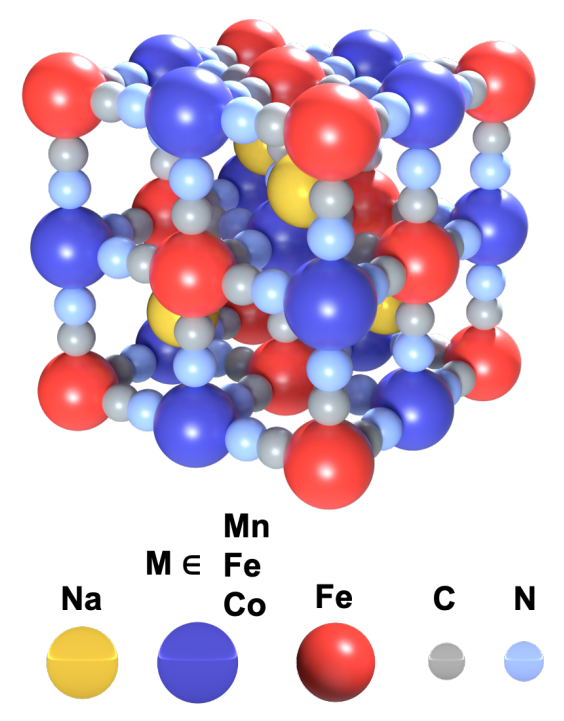

Figure 1: The PBA crystal structure. Two transition metals are octahedrally coordinated to cyanide ligands forming a cubic geometry (space group $\mathrm{Fm} \overline{3} \mathrm{~m}$ ) with wide channels for fast ionic conduction. Shown here is the intermediate oxidation state with one sodium ion (in the interstitial position) per formula unit. Application of an appropriate electrical potential results in oxidation (sodium removal) or reduction (sodium addition).

Studies on the effect of hydration state ${ }^{17}$ and nickel substitution at the nitrogen-coordinated site $^{18}$ gave similar values. The full range of values for the electronic conductivities of the PBAs remains unmeasured. Second, investigating their electronic properties using densityfunctional theory (DFT) is complicated because the PBAs are strongly correlated systems ${ }^{19}$ and DFT notoriously over-delocalizes $d$ orbital electrons in such systems. ${ }^{20}$ Early PBA models addressed the challenge with an ad hoc strategy of using different pseudopotentials for the two iron ions in the formula unit, ${ }^{21}$ and later work suggested that the composition $\mathrm{NaFe}\left[\mathrm{Fe}(\mathrm{CN})_{6}\right]$ is a half-metal. ${ }^{22,23}$ Most recently, the voltage profiles for numerous compositions were explored by Ong and coworkers, ${ }^{24}$ and Henkelman and coworkers used the nudged-elastic band method to understand ion diffusion in manganese hexacyanoferrate. ${ }^{25}$ The majority of current research uses DFT $+\mathrm{U} \cdot{ }^{26}$ Despite the success and usefulness of $\mathrm{DFT}+\mathrm{U}$, it requires the careful selection of the $\mathrm{U}$ parameter to match a calculated property to a value taken from experiment or from a more advanced calculation. This complicates its application to new, hypothetical PBAs, so a rigorous, purely ab initio strategy for modelling 
PBAs is desirable.

Here the electronic structure and electron-transport properties are explored systematically in three of the most promising metal hexacyanoferrates for sodium-ion cathodes: manganese hexacyanoferrate, $\left(\mathrm{Na}_{\mathrm{x}} \mathrm{Mn}\left[\mathrm{Fe}(\mathrm{CN})_{6}\right]\right)$, iron hexacyanoferrate $\left(\mathrm{Na}_{\mathrm{x}} \mathrm{Fe}\left[\mathrm{Fe}(\mathrm{CN})_{6}\right]\right)$, and cobalt hexacyanoferrate $\left(\mathrm{Na}_{\mathrm{x}} \mathrm{Co}\left[\mathrm{Fe}(\mathrm{CN})_{6}\right]\right)$. Each material is studied in each of three oxidation states $(\mathrm{x} \in\{0,1,2\})$ for a total of nine (9) compounds. The ground-state spin configuration for each compound is determined by comparing the total energy of the lowspin and high-spin phases. The projected density of states are calculated to examine the nature of the valence- and conduction-bands as well as to determine the compounds' band gaps. Finally, the charge-carrier effective masses are calculated. All the electronic properties are calculated within DFT employing either the generalized-gradient-approximation PerdewBurke-Ernzerhof (PBE) functional ${ }^{27}$ or the non-local, hybrid Heyd-Scuseria-Ernzerhof (HSE) functional. ${ }^{28}$

\section{Results and discussion}

\section{Ground-state spin configurations}

For PBAs, the carbon-coordinated metal ion assumes a low-spin (ls) electronic configuration while the nitrogen-coordinated metal is usually in a high-spin (hs) electronic configuration. In order to treat the spin configuration of PBAs within spin-polarized DFT calculations we assume a ferromagnetic ordering between the two metal centers and fix the overall spin, per formula unit, of the material, and perform a total energy calculation. Figure 2 shows the difference in total energy between the (ls, hs) configuration and the (ls, ls) configuration. Seven compounds are found to be more stable in the (ls, hs) configuration, and only compounds $\mathrm{Co}\left[\mathrm{Fe}(\mathrm{CN})_{6}\right]$ and $\mathrm{NaCo}\left[\mathrm{Fe}(\mathrm{CN})_{6}\right]$ are more stable in the (ls, ls) configuration. The total energies calculated using the HSE functional correctly reproduce the experimentally observed spin configuration for all nine compounds. ${ }^{1,2,13}$ Contrast these results with those calculated 
by the PBE functional, which incorrectly energetically favors the (ls, ls) configuration for eight of the nine compounds. All calculations results reported hereafter are performed within the DFT-HSE.
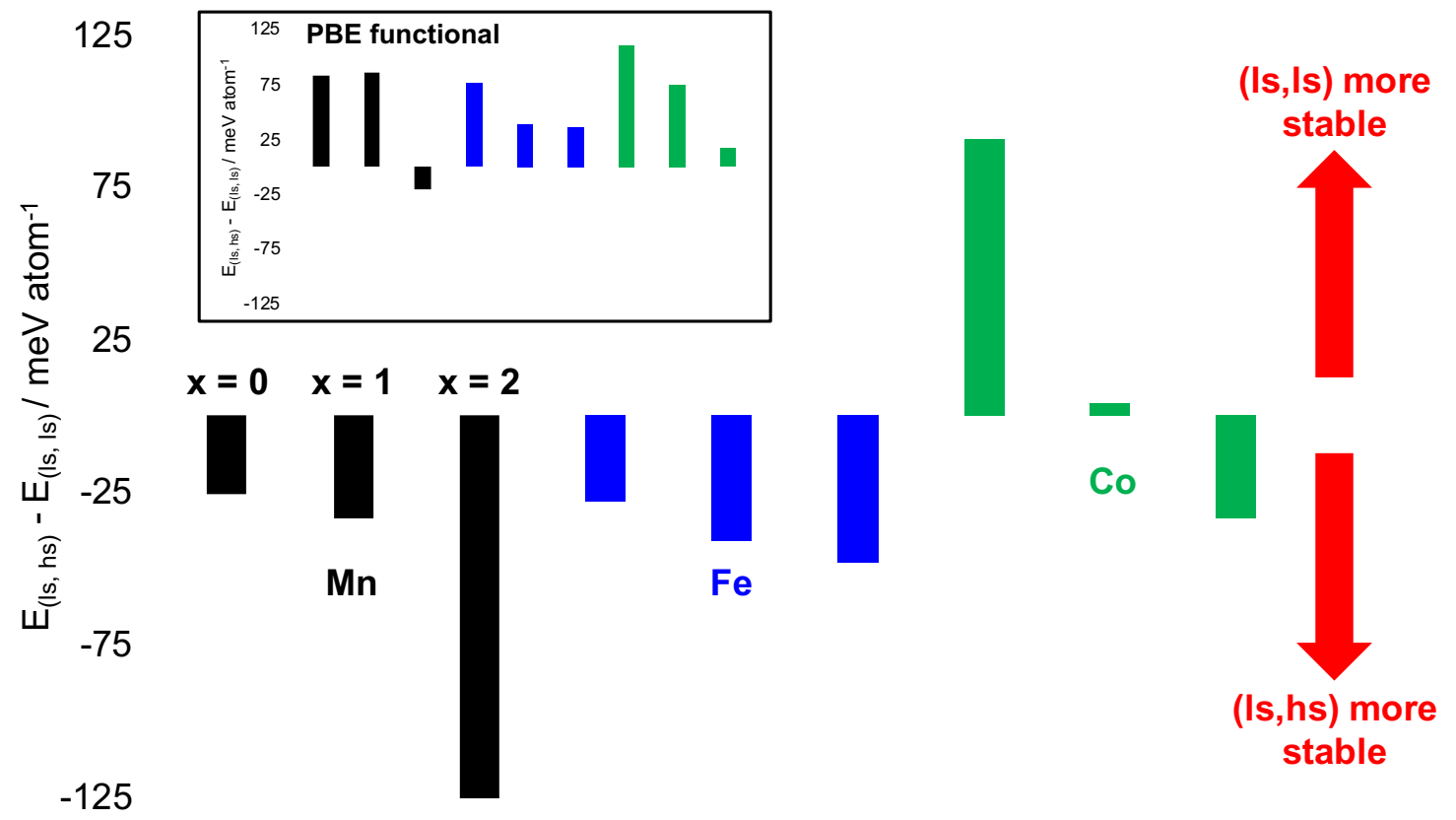

Figure 2: Energy differences between spin configurations. Negative differences correspond to energetic stability of the (ls, hs) state. Black, blue, and green bars correspond to $\mathrm{Mn}$, $\mathrm{Fe}$, and $\mathrm{Co}$, respectively. Within each color, the three bars represent 0,1 , or $2 \mathrm{Na}$-ions per formula unit from left to right. The inset shows the results using the PBE functional.

The compound $\mathrm{NaMn}\left[\mathrm{Fe}(\mathrm{CN})_{6}\right]$ can attain two $(\mathrm{ls}, \mathrm{hs})$ electronic configurations: $\left(\mathrm{Fe}\left(t_{2 g}^{5}\right)\right.$, $\left.\operatorname{Mn}\left(t_{2 g}^{3} e_{g}^{2}\right)\right)$ and $\left(\mathrm{Fe}\left(t_{2 g}^{6}\right), \operatorname{Mn}\left(t_{2 g}^{3} e_{g}^{1}\right)\right)$, with the latter lower in energy by less than $0.04 \mathrm{meV}$ atom $^{-1}$. These correspond to different oxidation states: (Fe(III), $\mathrm{Mn}(\mathrm{II})$ ) versus (Fe(II), $\mathrm{Mn}(\mathrm{III})$ ), respectively. Experiments have shown that below $225 \mathrm{~K}$, the $\left(\mathrm{Fe}\left(t_{2 g}^{6}\right), \operatorname{Mn}\left(t_{2 g}^{3} e_{g}^{1}\right)\right)$ phase is favored, in agreement with our DFT calculations; at higher temperatures, $\left(\mathrm{Fe}\left(t_{2 g}^{5}\right), \operatorname{Mn}\left(t_{2 g}^{3} e_{g}^{2}\right)\right)$ is observed. ${ }^{29}$ Furthermore, operando X-ray diffraction (XRD) ${ }^{30}$ and ex situ X-ray absorption near-edge structure (XANES) ${ }^{4}$ studies of room-temperature batteries clearly show that the manganese is first reduced from manganese(III) to manganese(II) and then the iron is reduced form iron(III) to iron(II). ${ }^{2}$ So in the rest of this work we only consider the technologically relevant $\left(\mathrm{Fe}\left(t_{2 g}^{5}\right), \mathrm{Mn}\left(t_{2 g}^{3} e_{g}^{2}\right)\right)$ phase of $\mathrm{NaMn}\left[\mathrm{Fe}(\mathrm{CN})_{6}\right]$. The three iron compounds are 
lower in energy in the $(\mathrm{ls}, \mathrm{hs})$ configuration. The intermediate oxidation state is $\left(\mathrm{Fe}_{\mathrm{C}}\left(t_{2 g}^{6}\right)\right.$, $\left.\mathrm{Fe}_{\mathrm{N}}\left(t_{2 g}^{3} e_{g}^{2}\right)\right)$, which is also consistent with experimental findings. ${ }^{1}$ The compounds' electronic configurations as calculated within HSE are shown in Figure 3.

\begin{tabular}{|c|c|c|c|c|}
\hline & & oxidatio & & duction \\
\hline & & $\mathrm{Na}_{0} \mathrm{M}\left[\mathrm{Fe}(\mathrm{CN})_{6}\right]$ & $\mathrm{Na}_{1} \mathrm{M}\left[\mathrm{Fe}(\mathrm{CN})_{6}\right]$ & $\mathrm{Na}_{2} \mathrm{M}\left[\mathrm{Fe}(\mathrm{CN})_{6}\right]$ \\
\hline Mn & (ls, hs) & $\overline{-}-e_{g}+\overline{-}$ & $\begin{array}{cc}-e_{g}+千 \\
+1+t^{t_{2 g}}+千+1\end{array}$ & $\begin{array}{c}-e_{g}+千+ \\
+\neq+t_{t_{2}}+千+1\end{array}$ \\
\hline & (Is, Is) & $\overline{+1-e^{e_{g}}-\bar{t}}$ & 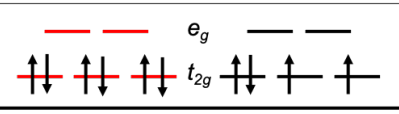 & 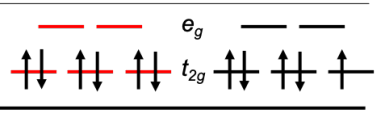 \\
\hline Fe & (Is, hs) & $\begin{array}{c}\overline{-} e_{g}+千+ \\
+1+t_{2 g}+千+1\end{array}$ & 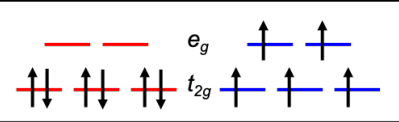 & 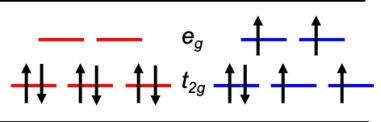 \\
\hline re & $(\mathrm{Is}, \mathrm{Is})$ & 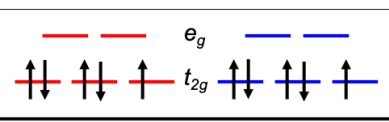 & 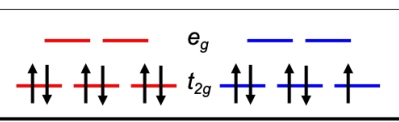 & 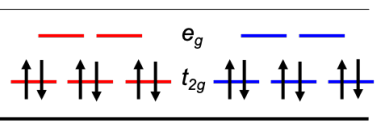 \\
\hline & (Is, hs) & 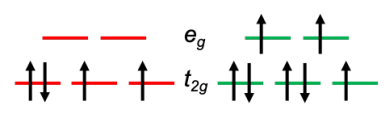 & $\begin{array}{cc}-e_{g}+\uparrow \\
\Downarrow+千 t_{2 g}+廿 \uparrow\end{array}$ & 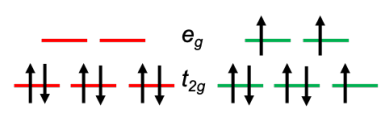 \\
\hline Co & $(\mathrm{Is}, \mathrm{Is})$ & 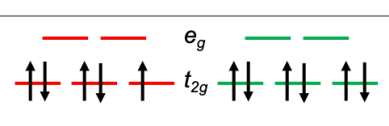 & 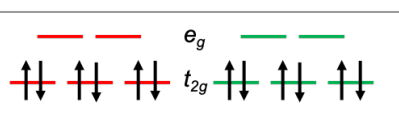 & 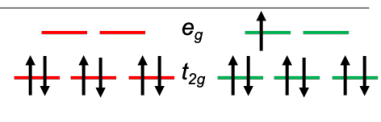 \\
\hline
\end{tabular}

Figure 3: Electronic configurations. The three $t_{2 g}$ orbitals and two $e_{g}$ orbitals are formed from the five $d$ orbitals of an octahedrally coordinated transition metal. The red orbitals correspond to carbon-coordinated iron, while black, blue, and green states refer to manganese, nitrogen-coordinated iron, and cobalt orbitals, respectively.

The two lower oxidation states of the cobalt compound (i.e. $\mathrm{Co}\left[\mathrm{Fe}(\mathrm{CN})_{6}\right]$ and $\left.\mathrm{NaCo}\left[\mathrm{Fe}(\mathrm{CN})_{6}\right]\right)$ are more stable in the (ls, ls) configuration; both contain cobalt(III, ls). On further reduction to $\mathrm{Na}_{2} \mathrm{Co}\left[\mathrm{Fe}(\mathrm{CN})_{6}\right]$, the cobalt becomes cobalt(II) and switches to high spin. This is in agreement with electrochemical observations of a spin transition over the second reduction plateau during battery discharge. ${ }^{31} \mathrm{NaCo}\left[\mathrm{Fe}(\mathrm{CN})_{6}\right]$ has the smallest energy difference between the two spin configurations at $4 \mathrm{meV}$ atom $^{-1}$. This small energetic difference is consistent with studies that observe facile photo-induced spin transition in this material. ${ }^{32}$ These experiments show that the spin transition is accompanied by a charge transfer from the iron to the cobalt. The calculated atom-projected magnetic moment vanishes on both the iron atom and the cobalt atom in the ground state, which is consistent with a non-spin- 
polarized electronic configuration of $t_{2 g}^{6}$ for iron and $t_{2 g}^{6}$ for cobalt. In the higher energy (ls,hs) state, the atom-projected magnetic moments are $\mu_{\mathrm{Fe}}=1.051 \mu_{\mathrm{B}}$ and $\mu_{\mathrm{Co}}=2.753 \mu_{\mathrm{B}}$ (where $\mu_{\mathrm{B}}$ is the Bohr magneton). This is consistent with an electronic configuration of $t_{2 g}^{5}$ for iron and $t_{2 g}^{5} e_{g}^{2}$ for cobalt, shown in Figure 3, and supports the experimental model of charge-transfer-accompanied spin transition. ${ }^{32}$ All other energy differences are greater (in magnitude) than the thermal excitation of $26 \mathrm{meV}$ atom $^{-1}$ at $300 \mathrm{~K}$.

In terms of crystal structure, the fully oxidized compound $\mathrm{Mn}\left[\mathrm{Fe}(\mathrm{CN})_{6}\right]$ has a tetragonal geometry. This is the result of a Jahn-Teller distortion arising from the electronic configuration on the manganese, $\operatorname{Mn}\left(t_{2 g}^{3} e_{g}^{1}\right)$. On reduction to $\operatorname{NaMn}\left[\mathrm{Fe}(\mathrm{CN})_{6}\right]$, the sodium ion occupies the center of the face separating two subcubes (i.e. the cubic interstice formed by eight cyanide ligands and four of each lattice metal ion), as shown in Figure SI-1. This relaxes the tetragonal distortion, but introduces a slight rhombohedral distortion. The lattice vectors are changed by about $2^{\circ}$ away from the value of $60^{\circ}$ for the face-centered cubic geometry. The fully reduced compound $\mathrm{Na}_{2} \mathrm{Mn}\left[\mathrm{Fe}(\mathrm{CN})_{6}\right]$ has its second sodium ion in a different face of the cube and is also rhombohedrally distorted. For the iron materials, the fully oxidized compound $\mathrm{Fe}\left[\mathrm{Fe}(\mathrm{CN})_{6}\right]$ is cubic. The reduced compounds $\mathrm{NaFe}\left[\mathrm{Fe}(\mathrm{CN})_{6}\right]$ and $\mathrm{Na}_{2} \mathrm{Fe}\left[\mathrm{Fe}(\mathrm{CN})_{6}\right]$ have their sodium ions in the cube faces and are rhombohedrally distorted. For the cobalt materials, $\mathrm{Co}\left[\mathrm{Fe}(\mathrm{CN})_{6}\right]$ is cubic and the reduced compounds are rhombohedrally distorted. For all compounds, the main difference between the (ls, ls) and the (ls, hs) configurations is a higher unit-cell volume for the (ls, hs) configuration and longer $\mathrm{M}_{\mathrm{N}}-\mathrm{N}$ bond length (where $\mathrm{M}_{\mathrm{N}}$ is $\mathrm{Mn}, \mathrm{Fe}$, or $\mathrm{Co}$ ). This is due to the increased density of spin-aligned electrons. Finally, comparing structural optimization between DFT-PBE with the hybrid HSE functional, we found that both result in basically the same geometry for all studied compounds.

These calculated geometries are consistent with two important phase changes that have been observed experimentally. First, fully desodiated $\mathrm{Mn}\left[\mathrm{Fe}(\mathrm{CN})_{6}\right]$ has been shown to be tetragonal. ${ }^{2}$ Second, $\mathrm{Na}_{\mathrm{x}} \mathrm{Mn}\left[\mathrm{Fe}(\mathrm{CN})_{6}\right], \mathrm{Na}_{\mathrm{x}} \mathrm{Fe}\left[\mathrm{Fe}(\mathrm{CN})_{6}\right]$, and $\mathrm{Na}_{\mathrm{x}} \mathrm{Co}\left[\mathrm{Fe}(\mathrm{CN})_{6}\right]$ all become 
rhombohedral above a critical sodium concentration between 1 and 2 sodium ions per formula unit, $1<\mathrm{x}_{\text {critical }}<2 .{ }^{33,34}$ Below $\mathrm{x}_{\text {critical }}$ the compounds are cubic (except for $\mathrm{Mn}\left[\mathrm{Fe}(\mathrm{CN})_{6}\right]$, which, as discussed, is tetragonal). The calculated geometries are already rhombohedral in the $\mathrm{x}=1$ phase. We attribute the early onset of the rhombohedral distortion to the fact that the simulated compounds are completely free of vacancies and crystalline water, both of which are known to stabilize the cubic structure over the rhombohedral structure. ${ }^{35}$ We show the simulated XRD patterns and crystal structures for the iron compounds in Figure SI.1.

\section{Atomic-projections of valence- and conduction-band states}

The projected density of states (PDOS) for the ground-state spin configuration of the nine materials as calculated with the HSE functional are shown in Figure 4. For $\mathrm{Mn}^{\mathrm{III}}\left[\mathrm{Fe}^{\mathrm{III}}(\mathrm{CN})_{6}\right]$, the valence-band states arise from spin-down iron $t_{2 g}$ orbitals, which are nearly degenerate with the spin-up manganese $e_{g}$ orbitals and cyanide orbitals. The conduction-band states are stem from spin-down, iron $t_{2 g}$ orbitals. The valence-band states for $\mathrm{NaMn}^{\mathrm{II}}\left[\mathrm{Fe}^{\mathrm{III}}(\mathrm{CN})_{6}\right]$ originate from the spin-up, manganese $e_{g}$ orbitals, in accordance with Figure 3 and the conduction-band states still arise from spin-down, iron $t_{2 g}$ orbitals. These become occupied on further reduction to $\mathrm{Na}_{2} \mathrm{Mn}^{\mathrm{II}}\left[\mathrm{Fe}^{\mathrm{II}}(\mathrm{CN})_{6}\right]$, in which the valence-band states stem from degenerate spin-up iron $t_{2 g}$ orbitals, spin-up manganese $e_{g}$ orbitals, spin-down iron $t_{2 g}$ orbitals, and cyanide orbitals. The conduction-band states originate from spin-down manganese $t_{2 g}$ orbitals and cyanide orbitals.

In $\mathrm{Fe}^{\mathrm{III}}\left[\mathrm{Fe}^{\mathrm{III}}(\mathrm{CN})_{6}\right]$, the valence-band states arise from spin-down, carbon-coordinatediron $t_{2 g}$ orbitals. The conduction band bottom stems from $t_{2 g}$ orbitals of both iron atoms, which is consistent with the $t_{2 g}^{5}$ and $t_{2 g}^{3}$ spin configuration shown in Figure 3. Upon reduction, $\mathrm{NaFe}^{\mathrm{III}}\left[\mathrm{Fe}^{\mathrm{II}}(\mathrm{CN})_{6}\right]$ also has its valence-band states originating from the carbon-coordinatediron, but the conduction-band states now solely originate from the nitrogen-coordinatediron $t_{2 g}$ orbitals. This can be explained due to the change of the carbon-coordinated-iron 


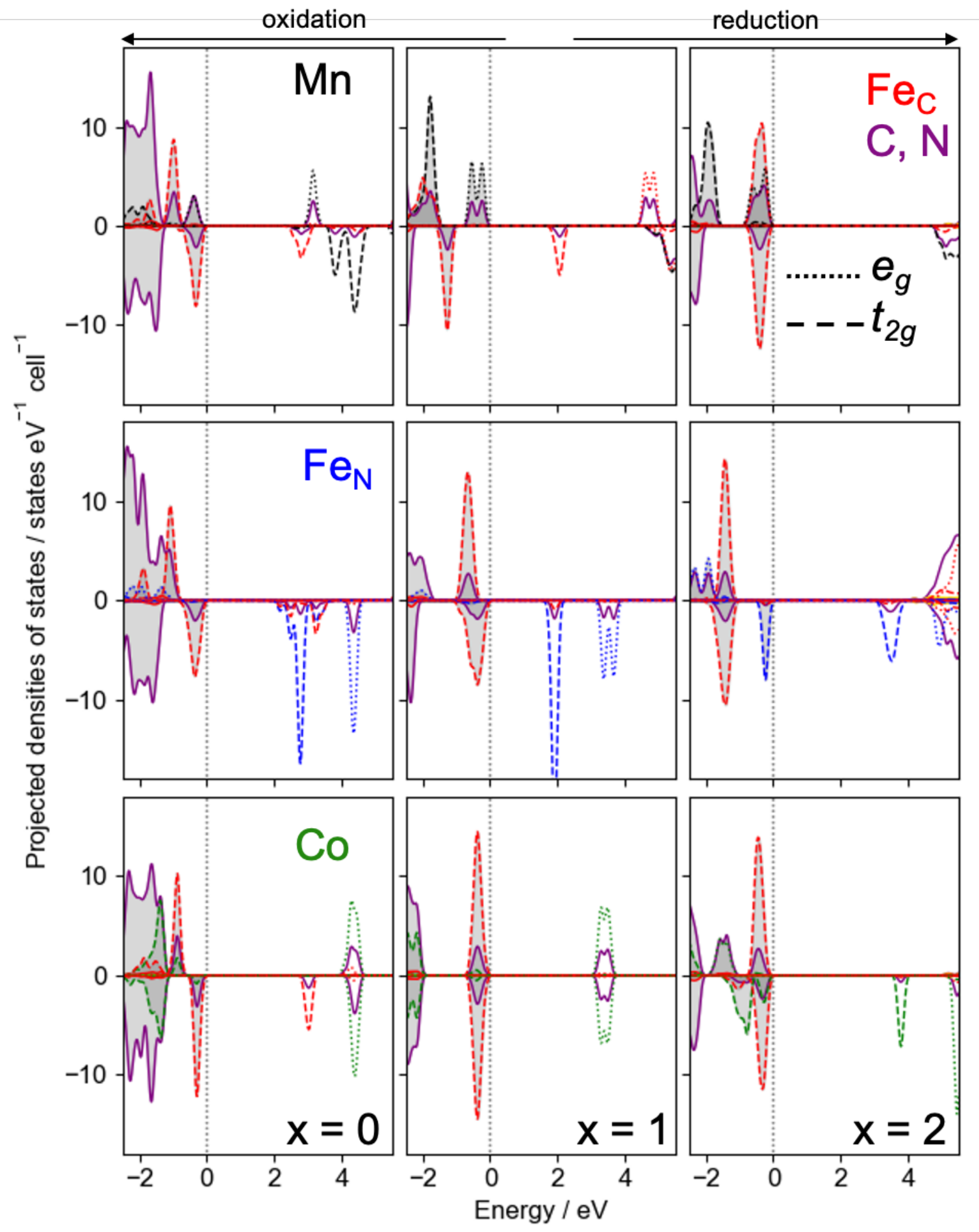

Figure 4: Projected density of states (PDOS) of the ground-state spin configuration to HSE. The top, middle, and bottom rows correspond to $\mathrm{Mn}, \mathrm{Fe}$, and Co, respectively. The left, middle, and right columns correspond to 0, 1, and 2 Na-ions per formula unit, respectively. The energy axis is referred to the Fermi level (gray, dotted line) and occupied states are shaded light gray. 
spin configuration to the fully filled $t_{2 g}^{6}$. Further reduction to $\mathrm{Na}_{2} \mathrm{Fe}^{\mathrm{II}}\left[\mathrm{Fe}^{\mathrm{II}}(\mathrm{CN})_{6}\right]$ breaks the symmetry of the spin-down nitrogen-coordinated-iron $t_{2 g}$ orbitals, one of which becomes occupied. Both the valence-band and the conduction-band states thus stem from spin-down orbitals.

Similarly in $\mathrm{Co}^{\mathrm{III}}\left[\mathrm{Fe}^{\mathrm{III}}(\mathrm{CN})_{6}\right]$, both the conduction- and valence-band states arise from spin-down, iron $t_{2 g}$ orbitals. For the reduced material, $\mathrm{NaCo}^{\mathrm{III}}\left[\mathrm{Fe}^{\mathrm{II}}(\mathrm{CN})_{6}\right]$, both Co- and Fe-sites are in the same fully filled $t_{2 g}^{6}$ spin configuration; hence, it is not spin-polarized. Valence-band states originate from the iron $t_{2 g}$ orbitals and the conduction-band states arise from cobalt $e_{g}$ orbitals (and some cyanide states). The spin transition of the cobalt on reduction to $\mathrm{Na}_{2} \mathrm{Co}^{\mathrm{II}}\left[\mathrm{Fe}^{\mathrm{II}}(\mathrm{CN})_{6}\right]$ changes the electronic structure substantially. The valenceband states stem from degenerate spin-up and spin-down iron $t_{2 g}$ orbitals. The valence band originate from spin-down cobalt $t_{2 g}$ orbitals.

In eight of the nine compounds either the valence-band or conduction-band states (or both) arise from $t_{2 g}$ orbitals of the carbon-coordinated iron. For all three fully oxidized materials $(\mathrm{x}=0)$, both the valence and conduction bands stem from the carbon-coordinated iron. This suggests that the carbon-coordinated iron participates significantly in the conduction of charge-carrying electrons and holes through the metal hexacyanoferrate lattice, especially for battery electrodes in the fully charged state. Sluggish kinetics caused by low electronic or ionic conductivity have been suggested as the cause of low coulombic efficiency, especially in manganese hexacyanoferrate. ${ }^{36}$ Research efforts to increase conductivity have focused on doping the nitrogen-coordinated element. ${ }^{4}$ However, if the low coulombic efficiency is caused by lower conductivity in the fully oxidized lattice, then these results suggest that experiment work must refocus on doping the carbon-coordinated iron to improve cycle lives of PBA batteries.

All nine materials have band gaps with values, as calculated with the HSE functional, shown in Table 1. The gaps range from $1.90 \mathrm{eV}$ to $4.94 \mathrm{eV}$. Even the smallest bang gap is too large for appreciable populations of thermally generated carriers at room temperature. The 
calculated band gap for Prussian blue, $\mathrm{NaFe}^{\mathrm{III}}\left[\mathrm{Fe}^{\mathrm{II}}(\mathrm{CN})_{6}\right]$, is $1.90 \mathrm{eV}$, versus an experimental value of $1.75 \mathrm{eV} .{ }^{37}$ The material $\mathrm{NaCo}\left[\mathrm{Fe}(\mathrm{CN})_{6}\right]$ has a direct band gap; the rest have indirect band gaps. PDOS calculated with the PBE functional are shown in Figure SI.2.

Table 1: Band gaps calculated using the HSE functional

\begin{tabular}{cccc}
\hline & $\mathrm{M}\left[\mathrm{Fe}(\mathrm{CN})_{6}\right]$ & $\mathrm{NaM}\left[\mathrm{Fe}(\mathrm{CN})_{6}\right]$ & $\mathrm{Na}_{2} \mathrm{M}\left[\mathrm{Fe}(\mathrm{CN})_{6}\right]$ \\
\hline $\mathrm{Mn}$ & 2.46 & 2.88 & 4.94 \\
$\mathrm{Fe}$ & 1.96 & 1.90 & 3.33 \\
$\mathrm{Co}$ & 3.00 & $3.33^{*}$ & 3.80 \\
\hline \multicolumn{4}{c}{${ }^{*}$ direct band gap }
\end{tabular}

\section{Charge-carrier effective masses and charge distributions}

The effective mass of an electron is defined as the second derivative of the electron energy with respect to the wavevector $\mathbf{k}$ about the minimum according to

$$
m_{i j}^{*}=\hbar^{2}\left(\partial^{2} \epsilon / \partial k_{i} \partial k_{j}\right)^{-1},
$$

in which $i$ and $j$ are the indices for the three crystallographic directions, $\epsilon$ is the energy eigenvalue, $\hbar$ is the reduced Planck's constant, and $m_{i j}^{*}$ is the $(i, j)$ component of the effective mass tensor. ${ }^{38}$ The partial derivatives are calculated numerically using a first-order, finitedifference approximation, and diagonalization of the effective-mass tensors gives the effective masses along the three principal axes. (The extremum $\mathbf{k}$ points of the valence-band tops and conduction-band bottoms are in Table SI.1 and 2, and the shapes of the energy isosurfaces near the extreme $\mathbf{k}$ points are shown in Figures SI.3 through 5). The isotropic average effective mass $\left(m^{*}=\left(\frac{1}{3} \Sigma_{i} m_{i}^{*-1}\right)^{-1}\right)$ for the compounds are given in Table SI.3. The hole effective masses range from $2.06 \mathrm{~m}_{\mathrm{e}}$ in $\mathrm{NaCo}\left[\mathrm{Fe}(\mathrm{CN})_{6}\right]$ to $3.62 \mathrm{~m}_{\mathrm{e}}$ in $\mathrm{Na}_{2} \mathrm{Mn}\left[\mathrm{Fe}(\mathrm{CN})_{6}\right]$ with a median value of $2.60 \mathrm{~m}_{\mathrm{e}}$ for all nine compounds. The electron effective masses range from $0.73 \mathrm{~m}_{\mathrm{e}}$ in $\mathrm{Fe}\left[\mathrm{Fe}(\mathrm{CN})_{6}\right]$ to $4.95 \mathrm{~m}_{\mathrm{e}}$ in $\mathrm{Co}\left[\mathrm{Fe}(\mathrm{CN})_{6}\right]$ with a median value of $1.94 \mathrm{~m}_{\mathrm{e}}$ for all nine compounds.

The effective masses are very heavy due to the fact that these compounds are ionic crystals, as shown in the band-decomposed charge densities for the valence and conduction 
bands of Figure 5. Eight of the nine compounds have significant valence-band charge density on the carbon-coordinated iron, and six of nine compounds have significant conductionband charge density on the carbon-coordinated iron. This is in agreement with the PDOS data, and underscores the need for investigation of the effect of doping at this site for tuning PBA electronic conductivity. Also of note is that the valence band in Prussian blue, $\mathrm{NaFe}\left[\mathrm{Fe}(\mathrm{CN})_{6}\right]$, is localized on the carbon-coordinated iron while the conduction band is localized on the nitrogen-coordinated iron. This is consistent with experimental observation of charge transfer between the two iron atoms on excitation of a valence electron to the conduction band. ${ }^{37}$ In general, the charge density is highly localized on the transition-metal ions. Together, the heavy effective masses and the ionic character of the electronic structure imply that small-polaron hopping is likely an important mechanism for charge conduction in these compounds. First-principles studies have shown the importance of small-polar hopping for electronic conductivity in battery materials including lithium iron phosphate ${ }^{39}$ and the (manganese, cobalt) oxide family. ${ }^{40}$ Tennakone and coworkers used small-polar hopping to interpret experimental data on the complicated relationship between crystalline water,

temperature, and electronic conductivity in Prussian blue $\left(\mathrm{NaFe}\left[\mathrm{Fe}(\mathrm{CN})_{6}\right]\right) .{ }^{17}$ These theoretical results rationalize Tennakone and coworkers' application of the small-polaron-hopping mechanism to understand their data; these results also call for future work to model and understand polarons in PBAs in greater detail.

\section{Conclusions}

Calculations from first principles using DFT to both the PBE- and HSE-level of theory identify important features of the electronic structure of three metal hexacyanoferrates in each of three oxidation states that are technologically relevant for next-generation battery cathodes. Comparison of the total energies of the (low spin, low spin) versus the (low spin, high spin) configurations as calculated by the HSE functional reproduces experimentally observed 

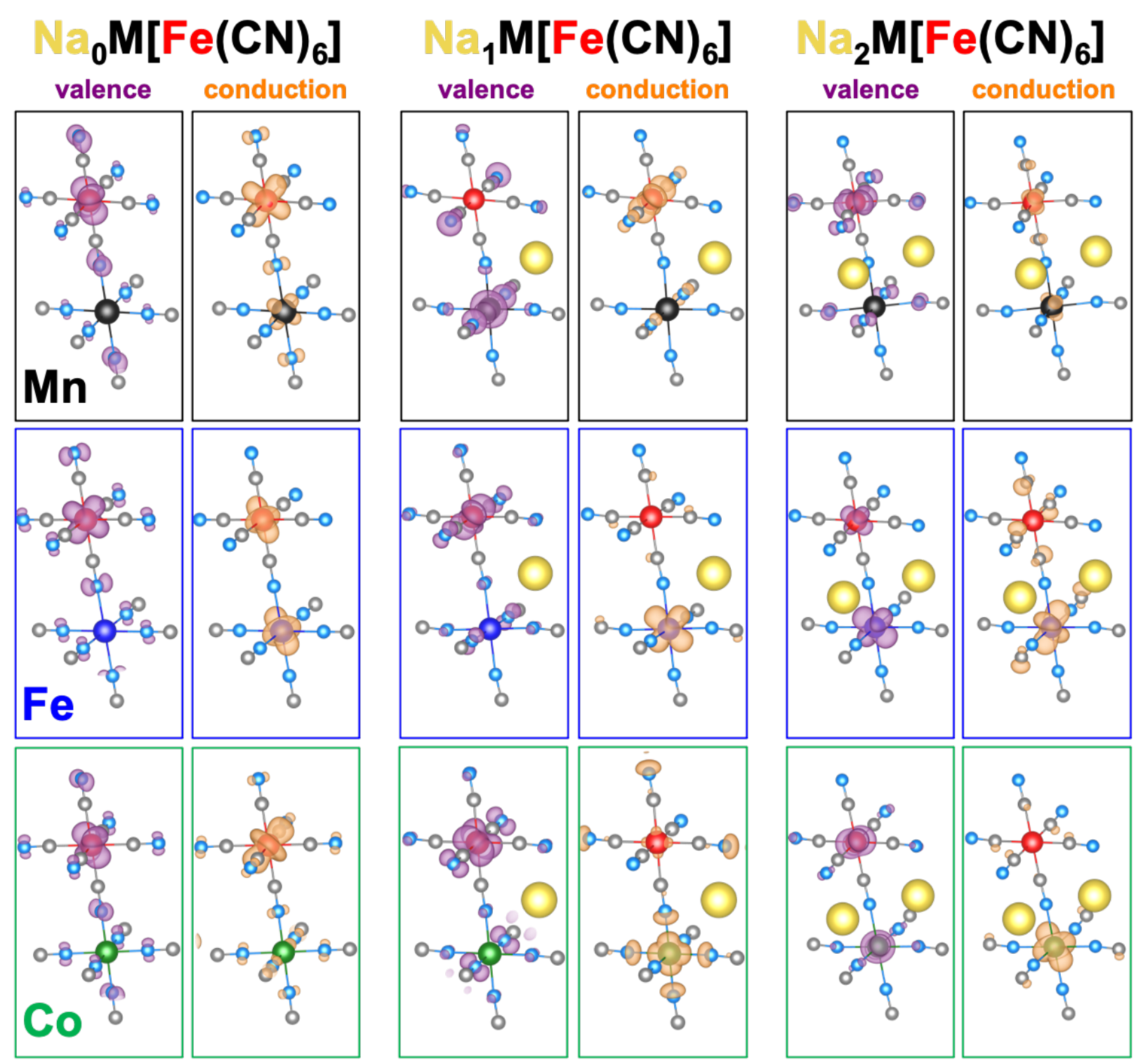

Figure 5: Band-decomposed charge densities. For each compound, the band-decomposed charge-density isosurface is plotted for the valence band (purple, left) and the conductionband (orange, right). The manganese, iron, and cobalt compounds are in the top, middle, and bottom rows, respectively. The number of sodium ions (gold) per formula unit are 0 (fully oxidized), 1, and 2 (fully reduced) from left to right. 
spin configurations in all nine compounds. This strategy provides a purely ab initio way of modelling PBA electronics. The PDOS and band decomposed charge density reveal the importance of the carbon-coordinated iron onto which conduction- and valence-bands states in many cases, confirming this as a promising site for substitutional doping to design more or less electronically conductive PBAs. The materials are all gapped with band gaps ranging from $1.90 \mathrm{eV}$ to $4.94 \mathrm{eV}$. The charge-carrier effective masses are calculated, with median values of $2.60 \mathrm{~m}_{\mathrm{e}}$ for holes and $1.94 \mathrm{~m}_{\mathrm{e}}$ for electrons. These very heavy effective masses, along with the highly ionic character of the compounds, suggest that small-polaron hopping may be an important mechanism for electron transport. Future work will center around modeling polarons in PBAs in greater detail. These results are important contributions to the study of PBA electronic conductivity and first-principles modeling, and this work lays the theoretical groundwork to incorporate electronic conductivity into the repertoire of tunable PBA properties.

\section{Methods}

All calculations were carried out using DFT as implemented in the Vienna ab inito Simulation Package (VASP) ${ }^{41,42}$ A $\Gamma$-centered $4 \times 4 x 4$ k-point grid and a plane-wave kinetic-energy cutoff of $520 \mathrm{eV}$ were used. Electronic and ionic convergence criteria were $10^{-5} \mathrm{eV}$ and $0.05 \mathrm{eV} \AA^{-1}$, respectively. Full geometric relaxation was performed on all materials with both the PBE and HSE functionals separately. For the HSE functional, we used a screening parameter of $0.2 \AA^{-1}$ and a mixing of $25 \%$ Fock exchange with $75 \%$ PBE exchange. The effective masses were calculated using a first-order finite-difference approximation of the second partial derivative of the energies at 18 zero-weight $\mathbf{k}$ points about the band extremum with a discretization of the local reciprocal space of $0.01 \cdot 2 \pi / a$. Band-decomposed charge densities were calculated using the band and $\mathbf{k}$ point that were used for the effective mass calculations. 


\section{Acknowledgement}

We gratefully acknowledge the use of the following computer resources: ARCHER UK National Supercomputing Service (http://www.archer.ac.uk); the University of Oxford Advanced Research Computing (ARC) facility (http://dx.doi.org/10.5281/zenodo.22558); and PRACE for awarding time on Beskow based in Sweden at KTH.

\section{Supporting Information Available}

Supplementary data are available online.

\section{References}

(1) Wang, L.; Song, J.; Qiao, R.; Wray, L. A.; Hossain, M. A.; Chuang, Y. D.; Yang, W.; Lu, Y.; Evans, D.; Lee, J. J.; Vail, S.; Zhao, X.; Nishijima, M.; Kakimoto, S.; Goodenough, J. B. Rhombohedral Prussian white as cathode for rechargeable sodium-ion batteries. J. Am. Chem. Soc. 2015, 13\%, 2548-2554.

(2) Song, J.; Wang, L.; Lu, Y.; Liu, J.; Guo, B.; Xiao, P.; Lee, J. J.; Yang, X. Q.; Henkelman, G.; Goodenough, J. B. Removal of interstitial $\mathrm{H}_{2} \mathrm{O}$ in hexacyanometallates for a superior cathode of a sodium-ion battery. J. Am. Chem. Soc. 2015, 137, 2658-2664.

(3) Wu, X.; Wu, C.; Wei, C.; Hu, L.; Qian, J.; Cao, Y.; Ai, X.; Wang, J.; Yang, H. Highly Crystallized Na2CoFe(CN)6 with Suppressed Lattice Defects as Superior Cathode Material for Sodium-Ion Batteries. ACS Appl. Mater. Inter. 2016, 8, 5393-5399.

(4) Jiang, L.; Lu, Y.; Zhao, C.; Liu, L.; Zhang, J.; Zhang, Q.; Shen, X.; Zhao, J.; Yu, X.; Li, H.; Huang, X.; Chen, L.; Hu, Y.-S. Building aqueous K-ion batteries for energy storage. Nat. Energy 2019, 
(5) Gao, H.; Xin, S.; Xue, L.; Goodenough, J. B. Stabilizing a High-Energy-Density Rechargeable Sodium Battery with a Solid Electrolyte. Chem 2018, 4, 833-844.

(6) Yu, Z.-Y.; Duan, Y.; Liu, J.-D.; Chen, Y.; Liu, X.-K.; Liu, W.; Ma, T.; Li, Y.; Zheng, X.S.; Yao, T.; Gao, M.-R.; Zhu, J.-F.; Ye, B.-J.; Yu, S.-H. Unconventional CN vacancies suppress iron-leaching in Prussian blue analogue pre-catalyst for boosted oxygen evolution catalysis. Nat. Commun. 2019, 10, 2799.

(7) Gao, C.; Yin, Y.; Zheng, L.; Liu, Y.; Sim, S.; He, Y.; Zhu, C.; Liu, Z.; Lee, H.-W.; Yuan, Q.; Lee, S. W. Engineering the Electrochemical Temperature Coefficient for Efficient Low-Grade Heat Harvesting. Adv. Funct. Mater. 2018, 1803129, 1803129.

(8) Shibata, T.; Moritomo, Y. Quick Response of All Solid Electrochromic Device. Appl. Phys. Express 2009, 2, 105502.

(9) Hurlbutt, K.; Wheeler, S.; Capone, I.; Pasta, M. Prussian Blue Analogs as Battery Materials. Joule 2018, 2, 1950-1960.

(10) Qian, J.; Wu, C.; Cao, Y.; Ma, Z.-F.; Huang, Y.; Ai, X.; Yang, H. Prussian Blue Cathode Materials for Sodium-Ion Batteries and Other Ion Batteries. Adv. Energy Mater. 2018, 1702619, 1-24.

(11) Wu, X.; Hong, J. J.; Shin, W.; Ma, L.; Liu, T.; Bi, X.; Yuan, Y.; Qi, Y.; Surta, T. W.; Huang, W.; Neuefeind, J.; Wu, T.; Greaney, P. A.; Lu, J.; Ji, X. Diffusion-free Grotthuss topochemistry for high-rate and long-life proton batteries. Nat. Energy 2019, 1.

(12) Pasta, M.; Wessells, C. D.; Huggins, R. A.; Cui, Y. A high-rate and long cycle life aqueous electrolyte battery for grid-scale energy storage. Nat. Commun. 2012, 3, 1149.

(13) Pasta, M.; Wang, R. Y.; Ruffo, R.; Qiao, R.; Lee, H.-W.; Shyam, B.; Guo, M.; Wang, Y.; Wray, L. A.; Yang, W.; Toney, M. F.; Cui, Y. Manganese-cobalt hexacyanoferrate cathodes for sodium-ion batteries. J. Mater. Chem. A 2016, 4, 4211-4223. 
(14) Scholz, F.; Dostal, A. The Formal Potentials of Solid Metal Hexacyanometalates. Angew. Chem. Int. Edit. 1996, 34, 2685-2687.

(15) Wang, C.; Chang, D.; Gao, Q.; Liu, C.; Wang, Q.; Huang, X.; Jia, Y. Large and tunable negative thermal expansion induced by a synergistic effect in $\mathrm{M}_{2}^{\mathrm{II}}\left[\mathrm{M}^{\mathrm{IV}}(\mathrm{CN})_{8}\right]$ Prussian blue analogues. Phys. Chem. Chem. Phys. 2020, 18655-18662.

(16) Xidis, A.; Neff, V. D. On the Electronic Conduction in Dry Thin Films of Prussian Blue, Prussian Yellow, and Everitt's Salt. J. Electrochem. Soc. 1991, 138, 3637-3642.

(17) Tennakone, K.; Dharmaratne, W. G. D. Experimental and theoretical study of electronic conduction in $\mathrm{H}_{2} \mathrm{O}$-doped Prussian blue. J. Phys. C Solid State 1983, 16, 56335639.

(18) Shrivastava, A.; Smith, K. C. Electron Conduction in Nanoparticle Agglomerates Limits Apparent $\mathrm{Na}^{+}$Diffusion in Prussian Blue Analogue Porous Electrodes. J. Electrochem. Soc. 2018, 165, A1777-A1787.

(19) Wojdel, J. C. First principles calculations on the influence of water-filled cavities on the electronic structure of Prussian Blue. J. Mol. Model. 2009, 15, 567-572.

(20) Himmetoglu, B.; Floris, A.; De Gironcoli, S.; Cococcioni, M. Hubbard-corrected DFT energy functionals: The $\mathrm{LDA}+\mathrm{U}$ description of correlated systems. Int. J. Quantum Chem. 2014, 114, 14-49.

(21) Wojdeł, J. C.; Bromley, S. T. From cluster calculations to molecular materials: A mixed pseudopotential approach to modeling mixed-valence systems. J. Mol. Model. 2005, 11, $288-292$.

(22) Wojdeł, J. C.; Moreira, I. D. P. R.; Bromley, S. T.; Illas, F. Prediction of half-metallic conductivity in Prussian Blue derivatives. J. Mater. Chem. 2009, 19, 2032. 
(23) Hegner, F. S.; Galán-Mascarós, J. R.; López, N. A Database of the Structural and Electronic Properties of Prussian Blue, Prussian White, and Berlin Green Compounds through Density Functional Theory. Inorg. Chem. 2016, 55, 12851-12862.

(24) Guo, X.; Wang, Z.; Deng, Z.; Li, X.; Wang, B.; Chen, X.; Ong, S. P. Water Contributes to Higher Energy Density and Cycling Stability of Prussian Blue Analogue Cathodes for Aqueous Sodium-Ion Batteries. Chem. Mater. 2019, 5933-5942.

(25) Xiao, P.; Song, J.; Wang, L.; Goodenough, J. B.; Henkelman, G. Theoretical study of the structural evolution of a $\mathrm{Na}_{2} \mathrm{FeMn}(\mathrm{CN})_{6}$ cathode upon $\mathrm{Na}$ intercalation. Chem. Mater. 2015, 27, 3763-3768.

(26) Ling, C.; Chen, J.; Mizuno, F. First-principles study of alkali and alkaline earth ion intercalation in iron hexacyanoferrate: The important role of ionic radius. J. Phys. Chem. C 2013, 117, 21158-21165.

(27) Perdew, J. P.; Burke, K.; Ernzerhof, M. Generalized Gradient Approximation Made Simple. Phys. Rev. Lett. 1996, 77, 3865-3868.

(28) Heyd, J.; Scuseria, G. E.; Ernzerhof, M. Hybrid functionals based on a screened Coulomb potential. J. Chem. Phys. 2003, 118, 8207-8215.

(29) Tokoro, H.; Ohkoshi, S. I.; Matsuda, T.; Hashimoto, K. A large thermal hysteresis loop produced by a charge-transfer phase transition in a rubidium manganese hexacyanoferrate. Inorg. Chem. 2004, 43, 5231-5236.

(30) Bie, X.; Kubota, K.; Hosaka, T.; Chihara, K.; Komaba, S. A novel K-ion battery: hexacyanoferrate(II)/graphite cell. J. Mater. Chem. A 2017, 5, 4325-4330.

(31) Kurihara, Y.; Funashima, H.; Ishida, M.; Hamada, N.; Matsuda, T.; Igarashi, K.; Tanida, H.; Uruga, T.; Moritomo, Y. Electronic structure of hole-doped transition metal cyanides. J. Phys. Soc. Jpn. 2010, 79, 1-7. 
(32) Cammarata, M.; Zerdane, S.; Balducci, L.; Azzolina, G.; Mazerat, S.; Exertier, C.; Trabuco, M.; Levantino, M.; Alonso-Mori, R.; Glownia, J. M.; Song, S.; Catala, L.; Mallah, T.; Matar, S. F.; Collet, E. Charge transfer driven by ultrafast spin transition in a CoFe Prussian blue analogue. Nat. Chem. 2021, 13, 10-14.

(33) You, Y.; Wu, X.-L.; Yin, Y.-X.; Guo, Y. G. High-quality Prussian blue crystals as superior cathode materials for room-temperature sodium-ion batteries. Energy Environ. Sci. 2014, 7, 1643-1647.

(34) Pramudita, J. C.; Schmid, S.; Godfrey, T.; Whittle, T.; Alam, M.; Hanley, T.; Brand, H. E.; Sharma, N. Sodium uptake in cell construction and subsequent in operando electrode behaviour of Prussian blue analogues, $\mathrm{Fe}\left[\mathrm{Fe}(\mathrm{CN})_{6}\right]_{1-x} \bullet y \mathrm{H}_{2} \mathrm{O}$ and $\mathrm{FeCo}(\mathrm{CN})_{6}$. Phys. Chem. Chem. Phys. 2014, 16, 24178-24187.

(35) Moritomo, Y.; Kurihara, Y.; Matsuda, T.; Kim, J. Structural phase diagram of Mn-Fe cyanide against cation concentration. J. Phys. Soc. Jpn. 2011, 80, 8-11.

(36) Fiore, M.; Wheeler, S.; Hurlbutt, K.; Capone, I.; Fawdon, J.; Ruffo, R.; Pasta, M. Paving the Way Toward Highly Efficient High-Energy Potassium-Ion Batteries with Ionic-Liquid Electrolytes. Chem. Mater. 2020, 32, 7653-7661.

(37) Robin, M. B. The Color and Electronic Configurations of Prussian Blue. Inorg. Chem. 1962, 1, 337-342.

(38) Filip, M. R.; Verdi, C.; Giustino, F. GW Band Structures and Carrier Effective Masses of $\mathrm{CH}_{3} \mathrm{NH}_{3} \mathrm{PbI}_{3}$ and Hypothetical Perovskites of the Type $\mathrm{APbI}_{3}: \mathrm{A}=$ $\mathrm{NH}_{4}, \mathrm{PH}_{4}, \mathrm{AsH}_{4}$, and $\mathrm{SbH}_{4}$. J. Phys. Chem. C 2015, 119, 25209-25219.

(39) Fisher, C. A.; Prieto, V. M.; Islam, M. S. Lithium battery materials $\operatorname{LiMPO}_{4}(M=$ $\mathrm{Mn}, \mathrm{Fe}, \mathrm{Co}$, and $\mathrm{Ni}$ ): Insights into defect association, transport mechanisms, and doping behavior. Chem. Mater. 2008, 20, 5907-5915. 
(40) Wang, Z. Q.; Chen, Y. C.; Ouyang, C. Y. Polaron states and migration in F-doped $\mathrm{Li}_{2} \mathrm{MnO}_{3}$. Phys. Lett. A 2014, 378, 2449-2452.

(41) Kresse, G.; Furthmüller, J.; Hafner, J. Theory of the crystal structures of selenium and tellurium: The effect of generalized-gradient corrections to the local-density approximation. Phys. Rev. B 1994, 50, 13181-13185.

(42) Kresse, G.; Furthmüller, J. Efficiency of ab-initio total energy calculations for metals and semiconductors using a plane-wave basis set. Comp. Mater. Sci. 1996, 6, 15-50. 
Graphical TOC Entry

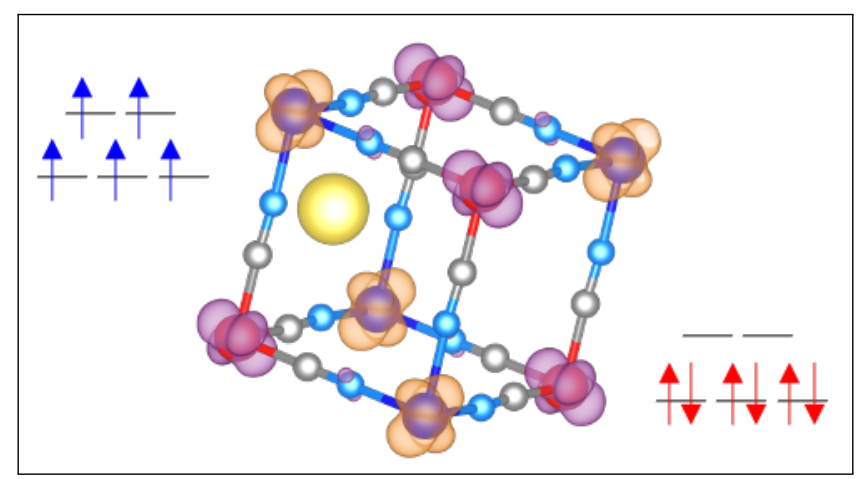

